\title{
3D Modeling of Transformer Substation Based on Mapping and 2D Images
}

\author{
Lei Sun, Xuesong Suo, Yifan Liu, Meng Zhang, and Lijuan Han \\ College of Mechanical \& Electrical Engineering, Agricultural University of Hebei, Baoding 071001, China \\ Correspondence should be addressed to Xuesong Suo; 13903120861@163.com
}

Received 27 January 2016; Revised 8 June 2016; Accepted 30 August 2016

Academic Editor: Mohammadreza Nasiriavanaki

Copyright (C) 2016 Lei Sun et al. This is an open access article distributed under the Creative Commons Attribution License, which permits unrestricted use, distribution, and reproduction in any medium, provided the original work is properly cited.

\begin{abstract}
A new method for building 3D models of transformer substation based on mapping and 2D images is proposed in this paper. This method segments objects of equipment in $2 \mathrm{D}$ images by using $k$-means algorithm in determining the cluster centers dynamically to segment different shapes and then extracts feature parameters from the divided objects by using FFT and retrieves the similar objects from 3D databases and then builds 3D models by computing the mapping data. The method proposed in this paper can avoid the complex data collection and big workload by using 3D laser scanner. The example analysis shows the method can build coarse 3D models efficiently which can meet the requirements for hazardous area classification and constructions representations of transformer substation.
\end{abstract}

\section{Introduction}

In recent years, substations under centralized management control mode has also been exposed to many problems with the increasing size of the grid, such as the following: the station complex and diverse equipment are too difficult to observe, the staff cannot quickly master station equipment environment [1-5]. Creating a visual system is a great help for the training of the operators. However, traditional workload of 3D modeling for transformer substation is too great, and the work period is too long.

In order to protect the security of staffs and facilitate their training, the 3D models of transformer substation by using laser scanning technology have increasingly aroused concerns of experts [6-9]. The rise of 3D laser scanning technology can solve the problem of the data acquisition and precision of the model while it may get a lot of points of information that leads to accurate modeling of the substation equipment [10-12]. However, the complex substation equipment structure will bring high point density by using 3D laser scanning technology which will increase the difficulty and time data collection and make the model work overload [13-15].

$3 \mathrm{D}$ reconstruction is one of the main research contents of the computer vision technology. In engineering and many other areas, in order to get useful information for research, we usually analyze the 3D structure of the objects. The research on $3 \mathrm{D}$ reconstruction has important practical significance and has a broad range of applications, such as archaeology, architecture, materials processing, industrial inspection, and medical imaging equipment industry [16-18].

In order to reduce the modeling workload, researchers have raised lots of 3D modeling methods by using 2D images [19-21]. Dr. Macro proposed a 3D reconstruction method by using a $2 \mathrm{D}$ detector under varying illumination conditions [22]. The method proposed by Dr. Macro is applied in head tracking which can extend the range of head motion and ensure the reconstruction fast and reliable. Literature [23] proposed a $3 \mathrm{D}$ modeling method based on $2 \mathrm{D}$ sketch. In literature [24] a reconstruction method by using new Zernike comparator is proposed which can provide a more accurate similarity measure, together with the optimal rotation, angle between the patterns, while keeping the same complexity as the classical approach. The method can ensure the automatic transcription of sketched storyboards into reconstructed 3D scenes. These method are more focused on 3D modeling based on texture map image; however the 3D modeling of transformer substation is more focused on the external 


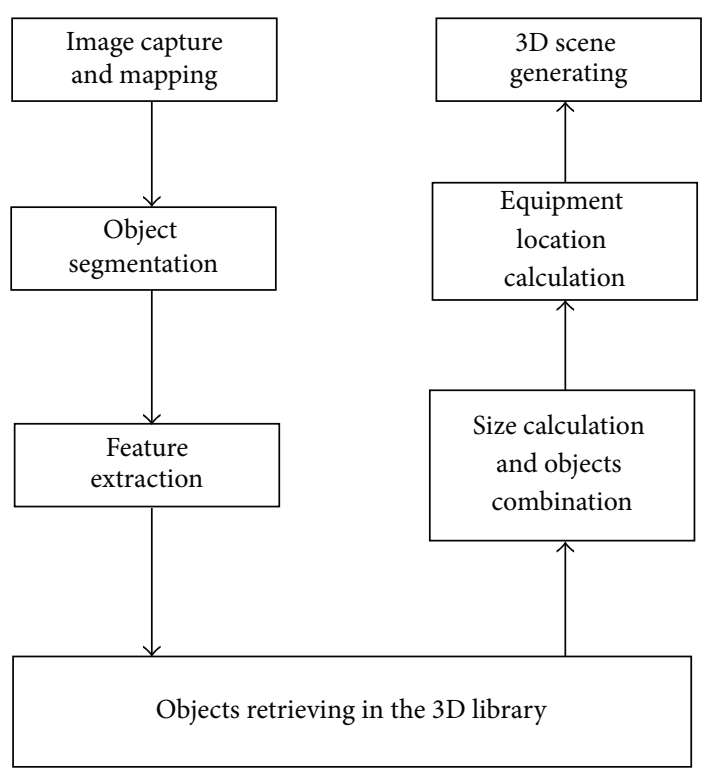

FIGURE 1: Flow chart of 3D modeling.

structure and shape of power equipment in order that staffs can zone security area and understand the environment of the substation easily $[25,26]$.

The method proposed in this paper is a $3 \mathrm{D}$ reconstruction technology based on 2D images which segments objects of equipment in $2 \mathrm{D}$ images to consist of a correspondingly shaped device model that is retrieved from the objects library. Then the size, spacing, and other parameters can be determined by the device mapping data. The 3D model established by this method not only can meet the requirements of zoning security, the substation shows, and so on, but also can reduce modeling costs and time greatly.

\section{Principle of the Modeling Method}

The difficulty of building 3D models from 2D images lies in lack of depth data. The method proposed in this paper takes shot from multiviews of equipment in the substation and then maps the position information of each device and gets equipment size, equipment location coordinates, and other information according to the scale. After making pixel division of the equipment, we retrieve the similar objects from the objects library by using the extracted features. The 3D size dimension of the objects is calculated according to the pixel in the image. Then determine the distance between the device and other aspects based on mapping data to establish the $3 \mathrm{D}$ modeling of the transformer substation. The user can also adjust the color and the concrete models. The process of establishing the 3D modeling is shown in Figure 1.

\section{Image Segmentation}

This paper sets sides of objects as $n$ side and $t$ side. We solve the energy function by using the improved $k$-means algorithm for cluster of different connection components, and then we can complete the image segmentation.

$s$ is the source point and $t$ is the terminal point, the connection side between $s$ and $t$ point is the $t$ side. $P$ and $q$ point are neighbor points, and the connection side between $p$ and $q$ point is the $n$ side.

We create a dynamic array $G=\left\{O_{0}, O_{1}, O_{2}, \ldots\right\}$ to store values of different segmentation shapes. Every side has a weight value $w$. Segmentation cost is defined as the summation weight value of all sides which is shown as

$$
C\left(O_{n}, O_{n+1}\right)=\sum_{p \in O_{n}, q \in O_{n+1}} w(p, q) .
$$

$(p, q)$ means the connection side between $p$ and $q$ point, $w(p, q)$ is the weight value of $(p, q), C\left(O_{n}, O_{n+1}\right)$ is the segmentation cost between the $n$th shape and $(n+1)$ th shape. We define the segmentation cost as the sign of the segmentation effect. The less the segmentation cost is, the better the segmentation effect is. Equation (2) is Gibbs function that can minimize the segmentation cost.

$$
E(X)=\sum_{p \in \delta} E_{1}\left(x_{p}, s \mid t\right)+\lambda \sum_{(p, q) \in \varepsilon} E_{2}\left(x_{p}, x_{q}\right) .
$$

As shown in (2), energy value function $E(X)$ consisted of the $t$ side energy values and $n$ side energy values. $E_{1}\left(x_{p}, s \mid\right.$ $t$ ) is the energy values of $t$ side which is the connection side between $s$ and $t$ point. $E_{2}\left(x_{p}, x_{q}\right)$ is the energy values of $n$ side which is the connection side between $p$ and $q$ point. $\lambda \geq 0$ is the parameter used to balance the two values. $x_{p}, x_{q}$ are pixels values of $p$ and $q$ point. $\delta$ is the set of points and $\varepsilon$ is the set of sides.

The $n$th cluster center is defined as $O_{n}$, and the Euclidean distance from one point to the $n$th cluster center is $d_{p}^{o_{n}}=$ $\min _{n}\left\|x_{p}-o_{n}\right\|$ and to the previous cluster center is $d_{p}^{o_{n-1}}=$ $\min _{n-1}\left\|x_{p}-o_{n-1}\right\|$. If the Euclidean distance is smaller, the point will be more similar to the previous cluster center. The Euclidean distance from point $p \notin\left\{O_{n}\right\} \cup\left\{O_{n-1}\right\}$ to the $n$th cluster center is shown as

$$
v=\frac{d_{p}^{o_{n}}}{d_{p}^{o_{n}}+d_{p}^{o_{n-1}}} .
$$

We can get $E_{1}\left(x_{p}, s \mid t\right)$ that is shown as

$$
\begin{array}{cccc} 
& p \in\left\{O_{n}\right\} & p \in\left\{O_{n-1}\right\} & p \notin\left\{O_{n}\right\} \cup\left\{O_{n-1}\right\} \\
E_{1}\left(x_{p}=1\right) & 0 & \infty & v \\
E_{1}\left(x_{p}=0\right) & \infty & 0 & 1-v
\end{array}
$$

According to above-mentioned equations and analytical result, we can get which energy values belong to $O_{n}$ and which 


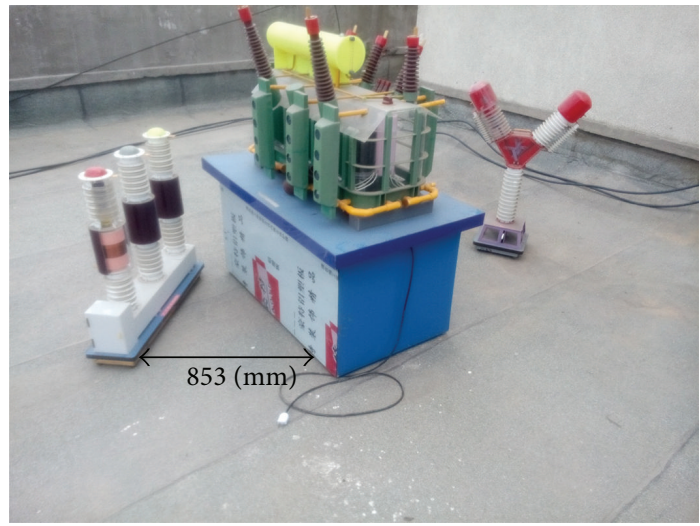

FIGURE 2: The 2D image of the sample.

energy values belong to $O_{n-1}$. If a point belongs to neither $O_{n-1}$ nor $O_{n}$, it will be defined as the $(n+1)$ th source point $s$ to repeat above process until all of the points are traversed.

For the $n$ side, we determine their respective section according to the relationship with the surrounding pixels. The more similar the $p$ and $q$ point are, the higher the value of $E_{2}$ is; on the contrary, the value of $E_{2}$ will tend to 0 . According to the color Euclidean distance between two pixels, we can define the energy value of $n$ side connection between the $p$ and $q$ point as

$$
E_{2}\left(x_{p}, x_{q}\right)=\frac{1}{\sqrt{2 \pi}} \exp \left(-\frac{\left\|x_{p}-x_{q}\right\|^{2}}{2}\right) \text {. }
$$

At last by solving the energy function we can determine the different outer contour shape and centroid of the objects, so that we can complete the image segmentation.

\section{Image Reconstruction}

This paper extracts feature parameters from the divided objects by using FFT. For pixels are discrete in the 2D image, we can descript the pixels in $N \times M$ image as

$$
\begin{aligned}
& f(x, y) \\
& =\left[\begin{array}{cccc}
f(0,0) & f(0,1) & \cdots & f(0, N-1) \\
f(1,0) & f(1,1) & \cdots & f(1, N-1) \\
\vdots & \vdots & \cdots & \vdots \\
f(M-1,0) & f(M-1,1) & \cdots & f(M-1, N-1)
\end{array}\right] .
\end{aligned}
$$

We set $N=M$ for convenience and then we can get discrete Fourier transform result as

$$
\begin{aligned}
F(u, v) & =\mathfrak{J}[f(x, y)] \\
& =\frac{1}{N} \sum_{x=0}^{N-1} \sum_{y=0}^{N-1} f(x, y) e^{-j 2 \pi((u x+v y) / N)} .
\end{aligned}
$$

Since the original image has been divided into simple 3D geometric objects that contain a small amount of information, setting $N=256$ can meet the requirement of retrieving. Then we can get $i$ th energy response as

$$
F_{i}=\left(\sum_{j=0}^{256} A_{j}\right)^{1 / 2}, \quad i=0,1,2, \ldots, 256 .
$$

Normalizing above equations we can get characteristic component of the target object as

$$
S_{F_{i}}=\frac{F_{i}}{\sum_{i=0}^{256} F_{i}} .
$$

Then we can the similarity degree between the retrieved object and the retrieval object by using Euclidean distance as

$$
S_{(q, d)}=\left(\sum_{i=0}^{255}\left(S_{F_{i} d}-S_{F_{i} q}\right)^{2}\right)^{1 / 2} .
$$

Then use the most similar objects after setting their $3 \mathrm{D}$ size to reconstruct the equipment.

\section{Case Analysis}

To verify the validity of the proposed method, we give an example of 3D model reconstruction analysis by combining objects. The $2 \mathrm{D}$ image of power equipment models is shown in Figure 2.

As mentioned above, objects shown in Figure 2 are composed of basic shapes. Segment Figure 2 as the method proposed in this paper, and we can get parts of objects as samples shown in Figure 3. In Figure 3 we can see that the basic shapes retrieved from the objects library are similar to the original basic shapes that can meet the requirements of building 3D transformer substation models.

By calculating according to the pixel in the image, we can get the 3D models of independent equipment as shown in Figure 4.

Then determine the distance between the device and other aspects based on mapping data to establish the $3 \mathrm{D}$ 

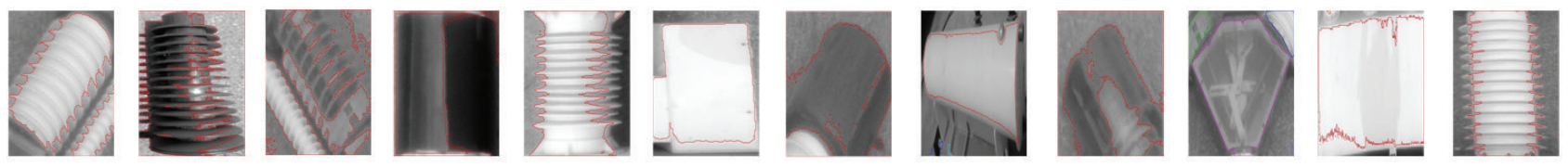<smiles>C1=CCCC1</smiles>
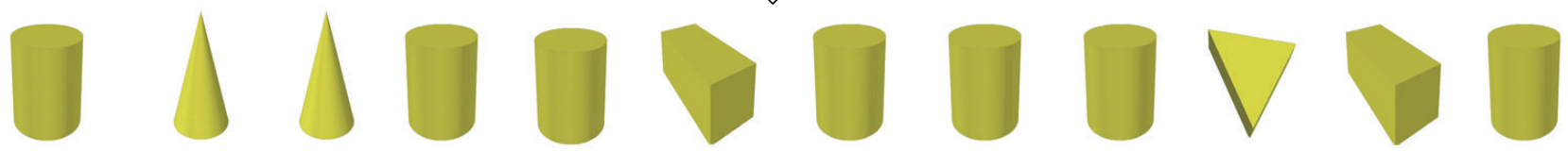

Figure 3: Parts of the divided objects.
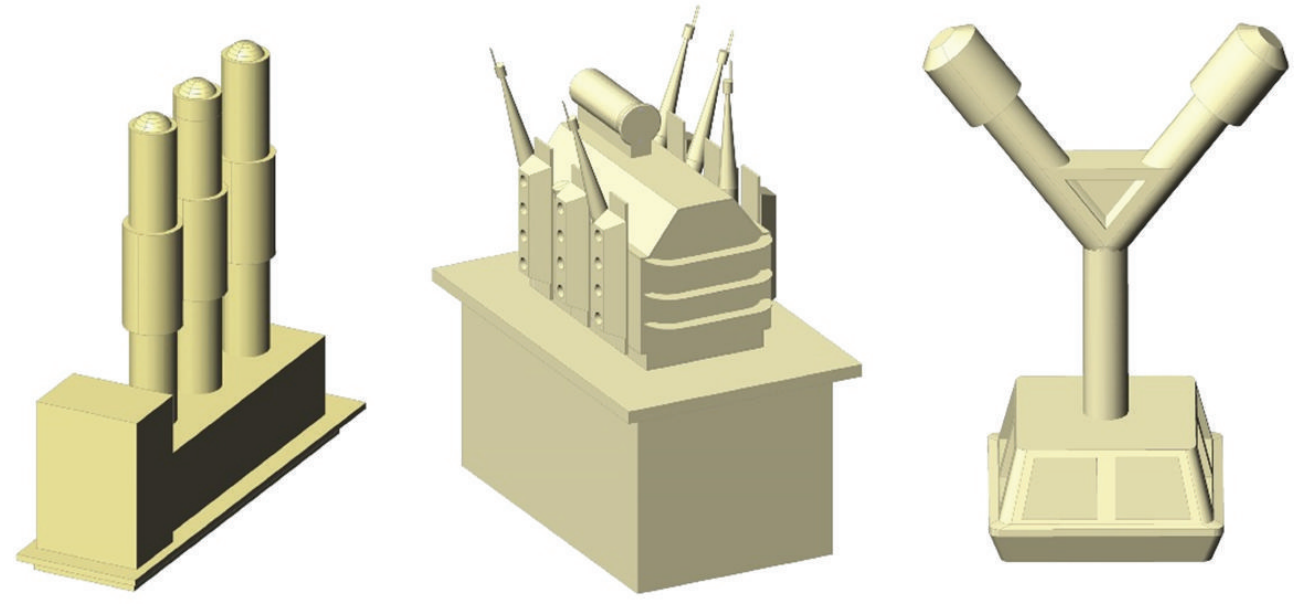

Figure 4: The 3D models image.

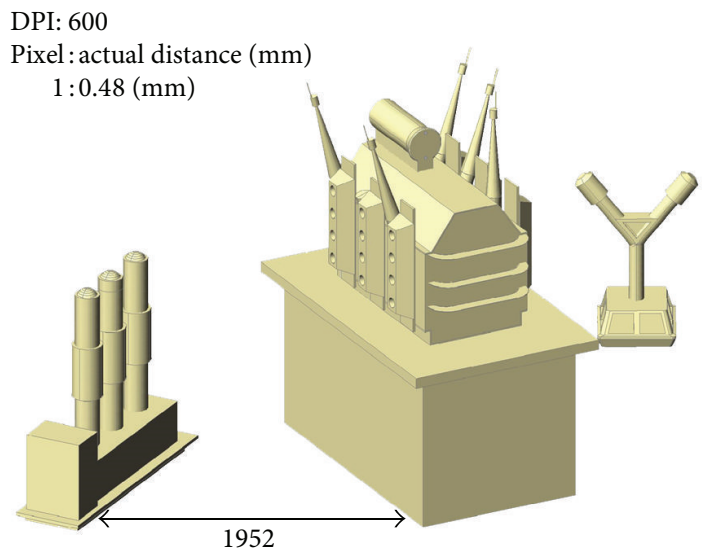

Figure 5: The 3D combination chart of the three power equipment.

model of the 2D image as shown in Figure 5. DPI of the image in Figure 5 is 600 , and the scale of it is a pixel to $0.48 \mathrm{~mm}$. The distance labeled in the image is 1952 pixels, and the distance converted by the scale is $937 \mathrm{~mm}$ while the actual distance shown in Figure 2 is $853 \mathrm{~mm}$. So the result of modeling can meet basic needs.
At last we built a 3D modeling of a transformer substation that its panorama, local view, and local enlarged view are shown as Figures 6, 7, and 8.

To verify the superiority of the method proposed in this paper, we make the curves of precision ratio-times as shown in Figure 9 by using three methods to retrieve a simple model. 


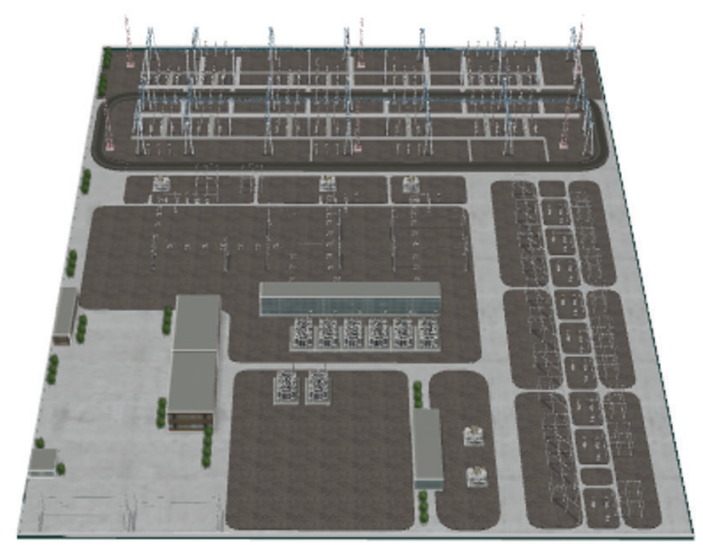

FIGURE 6: The 3D panorama of the transformer substation.

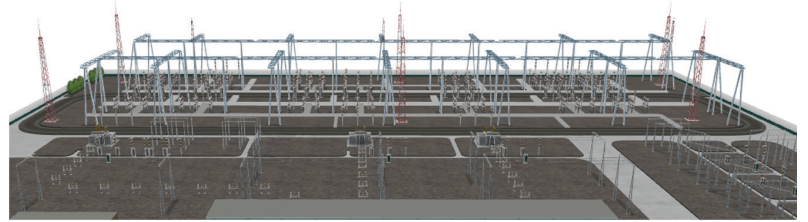

Figure 7: The 3D local view of the transformer substation.

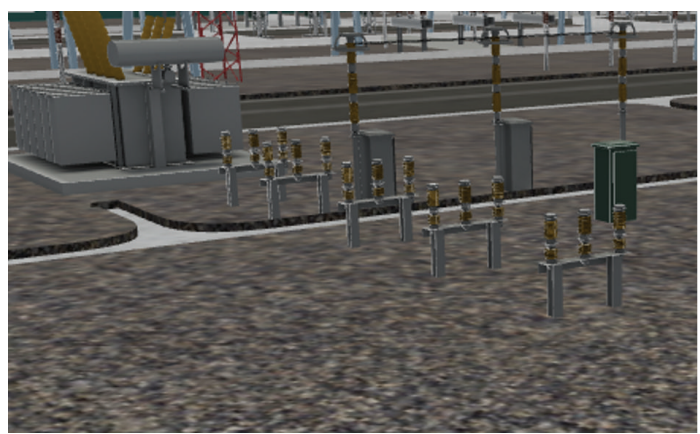

FIGURE 8: The 3D local enlarged view of the transformer substation.

As shown in Figure 9, the speed of 3D modeling method based on $2 \mathrm{D}$ sketch is the fastest while its precision ratio is lowest. 3D laser scanning technology can make the most accurate modeling; however it will increase the difficulty and time data collection and make the model work overload. The method proposed in this paper can achieve a high accuracy with a little time which can verify the validity of the method.

\section{Conclusions}

The diverse equipment in transformer station is too difficult to observe and the staff cannot quickly master station equipment environment. In order to protect the security of staffs and facilitate their training, this paper proposed a $3 \mathrm{D}$ modeling method based on mapping and 2D images. This method segments objects of equipment in $2 \mathrm{D}$ images by

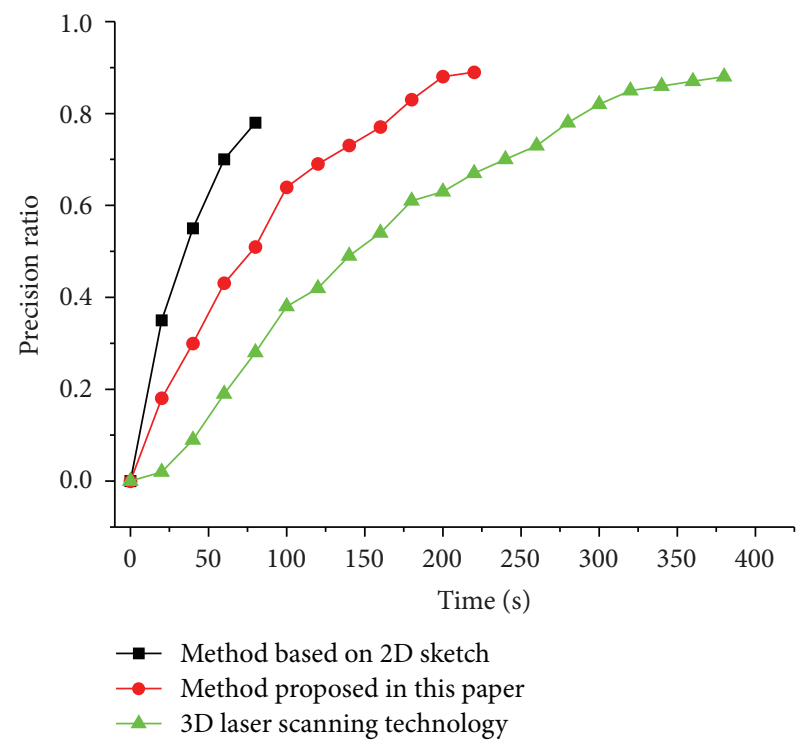

FIGURE 9: The precision ratio of three methods.

using $k$-means algorithm in determining the cluster centers dynamically to segment different shapes and then extracts feature parameters from the divided objects by using FFT and retrieves the similar objects from 3D databases and then builds $3 \mathrm{D}$ models by computing the mapping data. The $3 \mathrm{D}$ model established by this method not only can meet the requirements of zoning security, the substation shows, and so on, but also can reduce modeling costs and time greatly.

\section{Competing Interests}

The authors declare that they have no competing interests.

\section{Acknowledgments}

This work is supported by the project National High-Tech R\&D Program (863 Program) of China (2015AA050603). 


\section{References}

[1] H. Zhang, A. Chen, M. Niyi, and J. Ding, "Research on the key technology of smart substation model configuration and check," in Proceedings of the International Conference on Advanced Power System Automation and Protection (APAP '11), pp. 291294, October 2011.

[2] G. Romero, J. Maroto, J. Félez, J. M. Cabanellas, M. L. Martínez, and A. Carretero, "Virtual reality applied to a full simulator of electrical sub-stations," Electric Power Systems Research, vol. 78, no. 3, pp. 409-417, 2008.

[3] S.-Y. Park and M. Subbarao, "An accurate and fast point-toplane registration technique," Pattern Recognition Letters, vol. 24, no. 16, pp. 2967-2976, 2003.

[4] G. W. Yan, L. Zhang, and Y. F. Wang, "Research and implementation of the auto-generating system of three-dimensional substation simulation scene," in Proceedings of the IEEE International Conference on Intelligent Computing and Intelligent Systems (ICIS '10), pp. 768-771, IEEE, Xiamen, China, October 2010.

[5] D. Engel, C. Herdtweck, B. Browatzki, and C. Curio, "Image retrieval with semantic sketches," in Human-Computer Interaction-INTERACT 2011, vol. 6946 of Lecture Notes in Computer Science, pp. 412-425, Springer, Berlin, Germany, 2011.

[6] K. Xu, H. Zheng, H. Zhang, D. Cohen-Or, L. Liu, and Y. Xiong, "Photo-inspired model-driven 3D object modeling," ACM Transactions on Graphics, vol. 30, no. 4, article 80, 2011.

[7] Y. Liu, M. Zhou, and Y. Fan, "Using depth image in 3D model retrieval system," Advanced Materials Research, vol. 268-270, pp. 981-987, 2011.

[8] C. Goldberg, T. Chen, F.-L. Zhang, A. Shamir, and S.-M. $\mathrm{Hu}$, "Data-driven object manipulation in images," Computer Graphics Forum, vol. 31, no. 2, pp. 265-274, 2012.

[9] T. Lan, W. Yang, Y. Wang et al., "Image retrieval with structured object queries using latent ranking SVM," in Proceedings of the 12th European Conference on Computer Vision (ECCV '12), Florence, Italy, October 2012, vol. 7577 of Lecture Notes in Computer Science, pp. 129-142, Springer, 2012.

[10] J. Gaspar, Google Sketchup Pro 8 Step by Step, Vectorpro Publisher, 2011.

[11] S. Henrichs, "3ds max environment modeling\#1: procedural stone," 2010, http://saschahenrichs.blogspot.com.eg/2010/03/ 3dsmax-environment-modeling-1.html.

[12] J. W. H. Tangelder and R. C. Veltkamp, "A survey of content based 3D shape retrieval methods," Multimedia Tools and Applications, vol. 39, no. 3, pp. 441-471, 2008.

[13] D. Scharstein and R. Szeliski, "A taxonomy and evaluation of dense two-frame stereo correspondence algorithms," International Journal of Computer Vision, vol. 47, no. 1-3, pp. 7-42, 2002.

[14] R. Hartley and A. Zisserman, Multiple View Geometry in Computer Vision, Cambridge University Press, Cambridge, UK, 2nd edition, 2003.

[15] M. Z. Brown, D. Burschka, and G. D. Hager, "Advances in computational stereo," IEEE Transactions on Pattern Analysis and Machine Intelligence, vol. 25, no. 8, pp. 993-1008, 2003.

[16] O. Faugeras and Q.-T. Luong, The Geometry of Multiple Images, The MIT Press, Cambridge, Mass, USA, 2001.

[17] M. Pantic and L. J. M. Rothkrantz, "Automatic analysis of facial expressions: the state of the art," IEEE Transactions on Pattern Analysis and Machine Intelligence, vol. 22, no. 12, pp. 1424-1445, 2000 .
[18] C. Tomasi and T. Kanade, "Shape and motion from image streams under orthography: a factorization method," International Journal of Computer Vision, vol. 9, no. 2, pp. 137-154, 1992.

[19] N. Iyer, S. Jayanti, K. Lou, Y. Kalyanaraman, and K. Ramani, "Shape-based searching for product lifecycle applications," Computer Aided Design, vol. 37, no. 13, pp. 1435-1446, 2005.

[20] C. Migniot and F. Ababsa, "Hybrid 3D-2D human tracking in a top view," Journal of Real-Time Image Processing, vol. 11, no. 4, pp. 769-784, 2016.

[21] R. Ohbuchi and T. Furuya, "Scale-weighted dense bag of visual features for 3D model retrieval from a partial view 3D model," in Proceedings of the 12th IEEE International Conference on Computer Vision Workshops (ICCV '09), pp. 63-70, IEEE, Piscataway, NJ, USA, May 2009.

[22] M. L. Cascia, S. Sclaroff, and V. Athitsos, "Fast, reliable head tracking under varying illumination: an approach based on registration of texture-mapped 3D models," IEEE Transactions on Pattern Analysis and Machine Intelligence, vol. 22, no. 4, pp. 322-336, 2000.

[23] X. Xiaohua, "Three dimensional shape retrieval based on two dimensional skeches," Journal of Integration Technology, vol. 4, no. 2, pp. 22-33, 2015.

[24] J. Revaud, G. Lavoué, and A. Baskurt, "Improving Zernike moments comparison for optimal similarity and rotation angle retrieval," IEEE Transactions on Pattern Analysis and Machine Intelligence, vol. 31, no. 4, pp. 627-636, 2009.

[25] W. Rende, D. Yong, and S. Xiaojun, "Expectation and review on substation 3D modeling methods," North China Electric Power, vol. 2, pp. 19-23, 2015.

[26] W. Xianbing, Z. Xuedong, H. Tao et al., "Digital visualization management and monitoring system for 3D virtual transformer substations," Engineering Journal of Wuhan University, vol. 44, no. 6, pp. 786-791, 2011. 


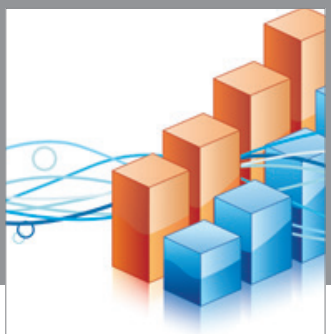

Advances in

Operations Research

vatem alat4

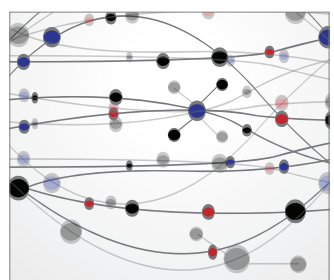

\section{The Scientific} World Journal
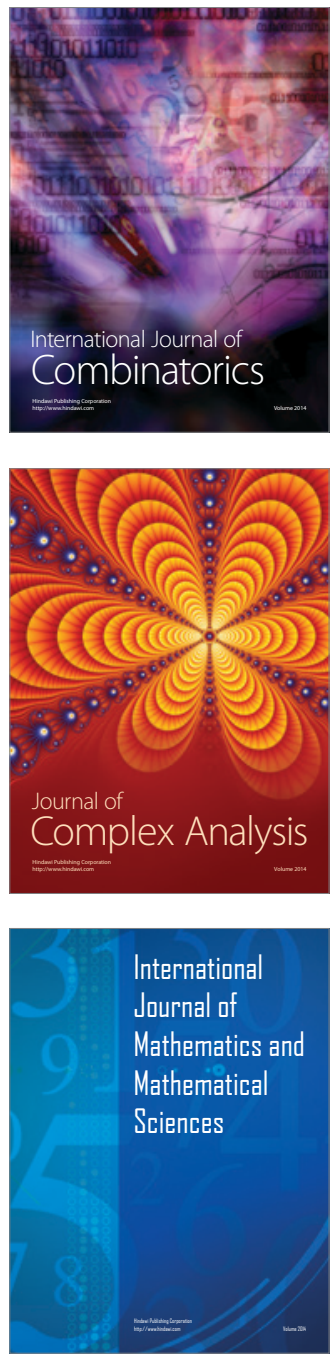
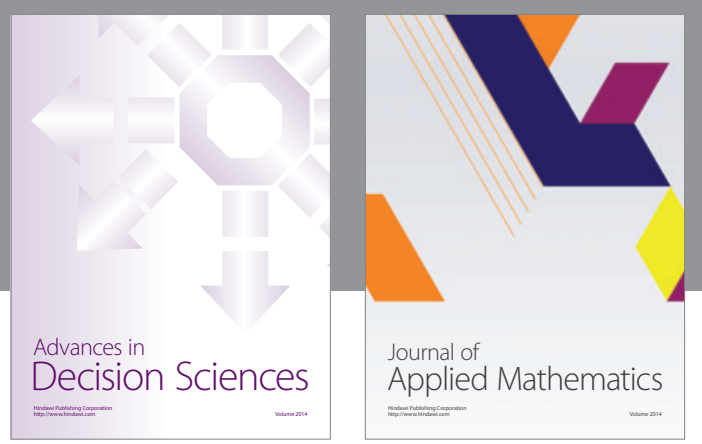

Algebra

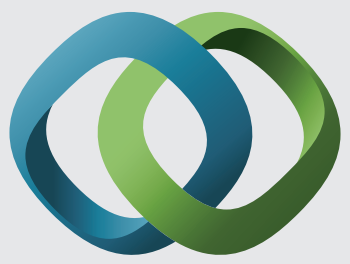

\section{Hindawi}

Submit your manuscripts at

http://www.hindawi.com
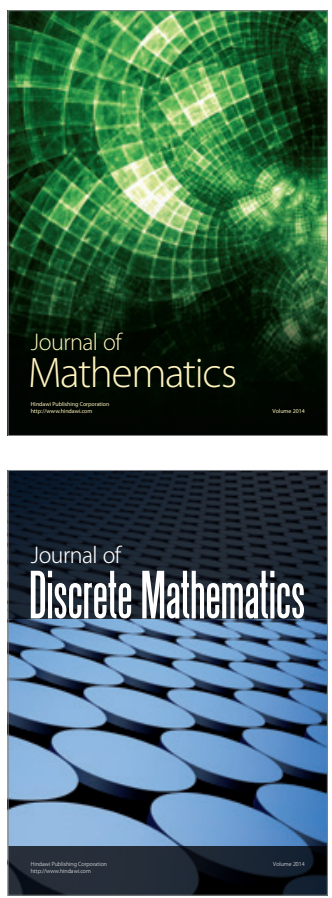

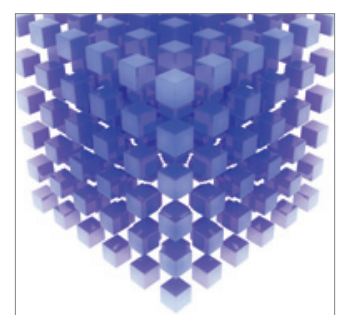

Mathematical Problems in Engineering
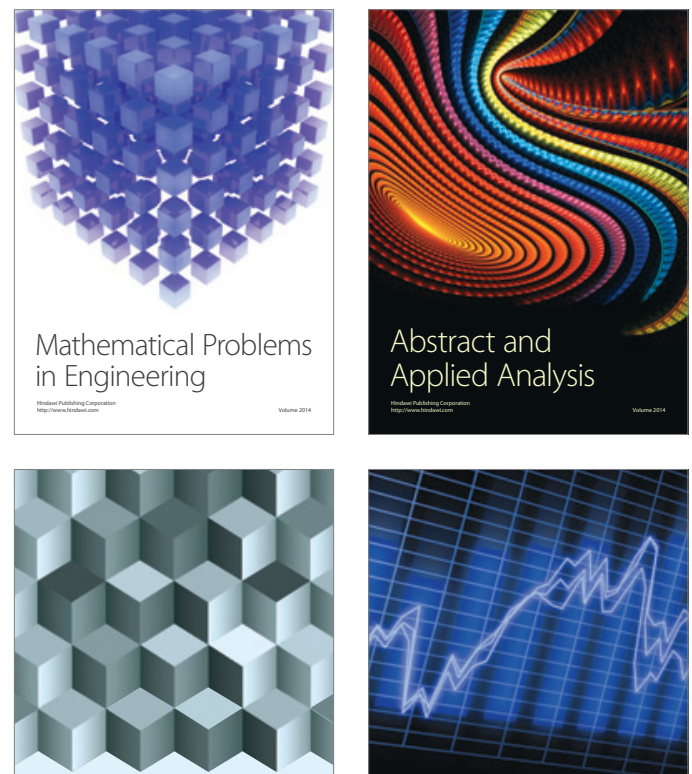

Journal of

Function Spaces

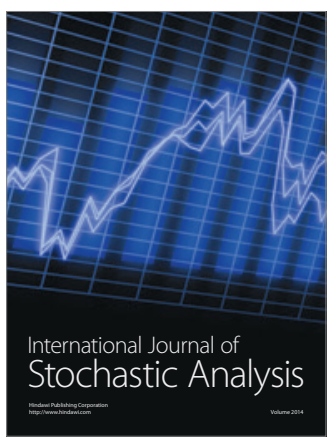

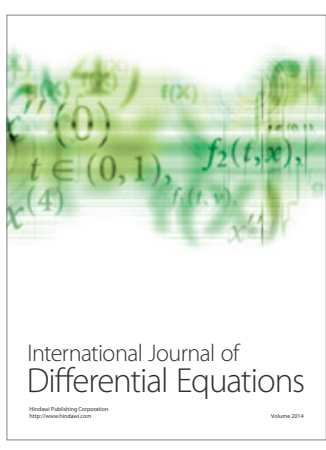
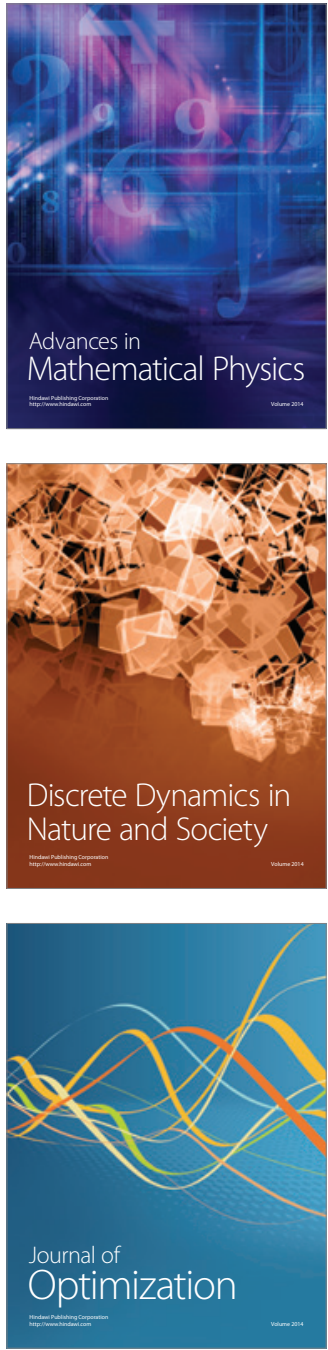